\title{
Single Photon Compressive Imaging Based on Digital Grayscale Modulation Method
}

\author{
Chenglong YUAN, Qiurong YAN*, Yiqiang WU, Yifan WANG, and Yuhao WANG
}

School of Information Engineering, Nanchang University, Nanchang 330031, China

${ }^{*}$ Corresponding author: Qiurong YAN

E-mail: yanqiurong@ncu.edu.cn

\begin{abstract}
In single-pixel imaging or computational ghost imaging, the measurement matrix has a great impact on the performance of the imaging system, because it involves modulation of the optical signal and image reconstruction. The measurement matrix reported in the existing literatures is first binarized and then loaded onto the digital micro-mirror device (DMD) for optical modulation, that is, each pixel can only be modulated into on-off states. In this paper, we propose a digital grayscale modulation method for more efficient compressive sampling. On the basis of this, we demonstrate a single photon compressive imaging system. A control and counting circuit, based on field-programmable gate array (FPGA), is developed to control DMD to conduct digital grayscale modulation and count single-photon pulse output from the photomultiplier tube (PMT) simultaneously. The experimental results show that the imaging reconstruction quality can be improved by increasing the sparsity ratio properly and compressive sampling ratio (SR) of these gray-scale matrices. However, when the compressive SR and sparsity ratio are increased appropriately to a certain value, the reconstruction quality is usually saturated, and the imaging reconstruction quality of the digital grayscale modulation is better than that of binary modulation.
\end{abstract}

Keywords: Single photon imaging; single pixel imaging; measurement matrix; grayscale modulation

Citation: Chenglong YUAN, Qiurong YAN, Yiqiang WU, Yifan WANG, and Yuhao WANG, "Single Photon Compressive Imaging Based on Digital Grayscale Modulation Method," Photonic Sensors, 2021, 11(3): 350-361.

\section{Introduction}

Single photon compressive imaging [1-3] is a technique using photon counting for ultra-weak light imaging based on the compressed sensing (CS) theory [4-7]. Single photon compressive imaging combines the advantages of single-pixel imaging [8-12], which can solve the problem of insufficient pixel size of existing single photon detectors and obtain higher imaging sensitivity. The ultra-high sensitivity of single photon compressive imaging can be achieved at the ultra-weak light level. Thus, single photon compressive imaging has broad application prospects in biomedical detection [13], spectral measurement [14], and 3-dimensional (3D) imaging [15-17].

The measurement matrix selected in the single photon compressive imaging system can obtain the relevant information of the image and determine the quality of image reconstruction [18, 19], which influence the performance of the imaging system. To study the better matrix performance of the measurement matrix in the single photon compressive imaging system, some researchers have designed some new measurement matrices in recent years. In 2015, Tiwari et al. [20] designed the sparse

Received: 03 December 2019 / Revised: 12 June 2020

(C) The Author(s) 2020. This article is published with open access at Springerlink.com DOI: $10.1007 / \mathrm{s} 13320-020-0597-y$

Article type: Regular 
sensing matrix to obtain high resolution medical images with least incurred computational cost. In 2017, based on Bernoulli and Gaussian random matrices, Nouasria et al. [21] constructed a new measurement matrix for compressed sensing by enhancing the orthogonality of the measurement matrix column. In 2018, Ding et al. [22] proposed a global perception matrix design method, which improved the performance of image compression in the multi-dictionary CS system.

The measurement matrix constructed by the above researchers based on compressed sensing theory improves the quality of image reconstruction. But in the single photon compressive imaging system, the measurement matrix loaded on digital micromirror device (DMD) is required to be a binary matrix, and the measurement matrix composed of 0 and 1 on the DMD contains less information, and if it is applied to gray image reconstruction, most of the information will be lost and it is difficult to ensure the accurate recovery of image information. The signal-to-noise ratio (SNR) is improved due to the large number of modulations of the gray matrix. Therefore, the modulation effect of the gray matrix is better than that of the binary matrix.

This paper proposes a digital grayscale modulation method, and constructs three different random measurement matrices with gray level through matrix transformation of different schemes. These matrices contain quaternary Gaussian random matrix, octal Gaussian random matrix, and hexadecimal Gaussian random matrix. Meanwhile, we construct a single photon compressive imaging system. In this system, the gray image is displayed by spatial light modulation of DMD and high precision timing control of field-programmable gate array (FPGA). Finally, we quantify the performance of the single photon compressive imaging system under different measurement matrices.

\section{Principle and realization of experimental system}

The single photon compressive imaging system is constructed as shown in Fig. 1. The designed single photon source of ultra-weak parallel light can reduce the effect of light source scattering on imaging results. The target image is illuminated by ultra-weak parallel light and then imaged on the DMD by imaging lens. The DMD used in the experiment is composed of $1024 \times 768$ micromirror arrays, and each micromirror can independently realize $\pm 12^{\circ}$ deflection and it can modulate the input light under the control of measurement matrix loaded on the DMD. Under the control of the synchronous control module, the DMD selects a part of the micromirror to flip $+12^{\circ}$ for each spatial synchronization of the optical image. Using a photomultiplier tube (PMT) photon counting head as the single photon detector, the modulated light is collected into a single photon detector through focusing lens set along the $+12^{\circ}$ reflecting direction of the micromirror.

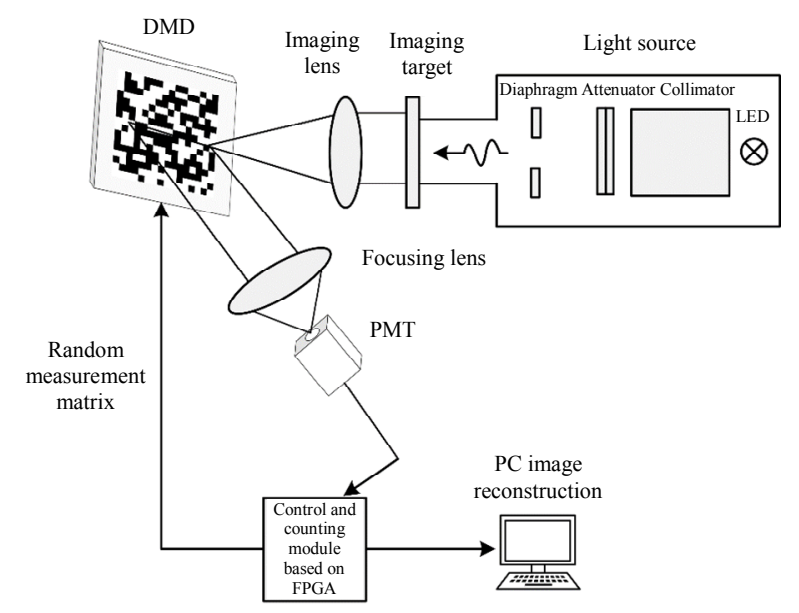

Fig. 1 Schematic diagram of a single photon compressive imaging system, including a parallel single photon source (it consists of light emitting diode (LED) lamp, collimator, attenuator, and diaphragm), imaging target, imaging lens, DMD, focusing lens, and PMT.

The system adopts the idea of compressed sensing for compressed sampling and image reconstruction. The modulation process of each 
frame matrix for the optical image on the DMD can be regarded as the inner product between the measurement matrix on the DMD and the original image. The inner product is the number of photons detected in the direction of reflection of $+12^{\circ}$ during each measurement time. The measurement process is carried out under the synchronous control module based on the FPGA. In the experiment, the measurement matrix is loaded sequentially by the FPGA to the DMD for modulating the image, and synchronously records the number of photons output by a photon counting photomultiplier tube (Hamamatsu Photonics H10682-110 PMT) with an effective photosensitive area of $\Phi 8 \mathrm{~mm}$. And the number of photons is proportional to the intensity of light. Finally, the image is reconstructed by the compressed sensing algorithm according to the measurement matrix loaded to the DMD and the photon sequence $y$ is received by the synchronization control module.

Let the image $x$ be a one-dimensional signal of length $N$ and the sparsity $K$ (i. e., containing $K$ non-zero values). The mathematical model of the above compression measurement process can be expressed as follows:

$$
y=\boldsymbol{\Phi} x+e .
$$

In the formula, $e$ is the measurement noise, and $\Phi$ is the measurement matrix of the $M \times N$ dimension, and $M$ is much smaller than $N$. We can reconstruct the image $x$ from the obtained $M$-dimensional measured values. The measured value $y$ is an $M$-dimensional vector, which reduces the measurement object from $\mathrm{N}$-dimensional to $M$-dimensional. The design of the measurement matrix requires that the measured $M$ measurements cannot destroy the original signal during the conversion of the signal from $x$ to $y$, ensuring that the signal can be reconstructed accurately. When $M<N$, there are infinite solutions and the signals cannot be reconstructed in theory. However, the original image $x$ is $k$-sparse or sparse on the orthogonal sparse basis $\Psi$ and $x$ will have a greater probability of being reconstructed from a small number of measurements $y$. That is $x=\Psi \alpha$, and $\alpha$ is a sparse signal with a number of non-zero elements much smaller than $N$, then (1) can be rewritten as:

$$
y=\boldsymbol{\Phi} \Psi \alpha+e .
$$

Defined as $\mathbf{A}^{\mathrm{CS}}=\boldsymbol{\Phi} \Psi, \mathbf{A}^{\mathrm{cs}}$ is the matrix of $M \times N$. The reconstruction of image $x$ is usually done by solving the $L_{1}$ norm. The reconstruction process of compressed sensing can be described by the following equation:

$$
\hat{x}=\arg \min _{x \in R^{N}}\|\alpha\|_{1}, \text { s.t. } y=\mathbf{A}^{\mathrm{CS}} \alpha+e
$$

where $x$ is the original image. In the theory of compressed sensing, there are many algorithms for solving (3). The greedy algorithm directly based on the $L_{0}$ norm solution [such as matching pursuit (MP) and orthogonal matching pursuit (OMP)] converts $L_{0}$ into $L_{1}$ relaxation algorithm of $L_{1}$ problem [such as base tracking (basis pursuit, BP)] and total variation algorithm [such as total variation augmented lagrangian alternating direction algorithm, TVAL3)] [23, 24]. In this paper, combining the performance of various algorithms, the TVAL3 algorithm is used as the reconstruction algorithm of the target image, and it has lower complexity and faster reconstruction.

\section{Implementation of DMD digital gray matrix modulation}

It has been proved theoretically and practically that matrices satisfying the restricted isometry property (RIP) can be used as the compressed sensing measurement matrix. Bernoulli and Gaussian matrices are proposed to satisfy restricted isometry property with high probability and have the advantages of a simple structure and a high reconstruction efficiency [25-27]. At present, the Gaussian random measurement matrix is generally transformed into $0-1$ binary matrix and applied to the DMD, which can modulate the simple binary image. But when applied to gray-level images, the 
binary matrix carries less information and increases the computational complexity in the observation process and has a great impact on the recovery accuracy. If the Gaussian random measurement matrix is converted into a corresponding gray matrix, the same imaging effect can be achieved correspondingly, and the reconstruction accuracy of the gray image is also improved.

\subsection{Construction of the digital gray-scale matrix}

Firstly, this scheme generates a matrix $\boldsymbol{\Phi}$ of $M \times N$, and makes every element between 0 and 1 in matrix $\boldsymbol{\Phi}$ obey the Gaussian distribution with the mean value of 0 and the variance of $1 / M$ independently.

$$
\boldsymbol{\Phi}_{i, j} \sim N\left(0, \frac{1}{M}\right) .
$$

The measurement matrix has strong randomness. It can be proved that when the number of measurements of the Gaussian random matrix is $M \geq c K \lg (N / K)$, the RIP condition is satisfied with a maximum probability [28]. Gaussian random matrix is widely used in CS, mainly because it is not related to most orthogonal basis or orthogonal dictionary. At the same time, because of its simple structure and it is easy to be constructed, the image can be accurately reconstructed from a small amount of measured data.

Then, each element in the Gaussian matrix constructed is converted into a measurement matrix whose matrix element values are independently distributed between 0 and 3, 0 and 7, and 0 , and 15 according to corresponding coefficients of a certain proportion. Finally, the values in the matrix are rounded and converted into the corresponding matrix elements of Gaussian random matrices in quaternary, octal, and hexadecimal, respectively.

\subsection{Implementation of DMD grayscale modulation}

In this paper, the method of DMD grayscale modulation is applied to the single photon compressive imaging system for the modulation of gray matrix, and the binary pulse width modulation (PWM) method is used to realize the full digital control of the image gray level by the DMD. PWM uses binary and division of time pulse width modulation technology to process binary weighted image data. The length of the pulse width indicates the reflection time of the position of the micromirror to determine the magnitude of the gray value at that location, so as to control the time of a single micromirror switch to form the gray modulation of each pixel of the image [29,30]. The method of grayscale display based on the DMD adopted in this paper is the same as accessory light modulator package (ALP) in principle [31]. By this method, the input image signal of the DMD can be converted into gray image with rich levels. Binary PWM modulation can be expressed as follows:

$$
T_{\mathrm{PWM}}=T_{\mathrm{LSB}} 2^{N-1}
$$

where $N$ is the gray level of the pixel, $T_{\mathrm{PWM}}$ is the weight of each bit pixel, and $T_{\mathrm{LSB}}$ is the display time of the lowest bit (LSB).

As shown in Fig. 2, it is an example of a pulse sequence controlled by PWM for 4-bit binary image data with 10 gray values. It has $2^{4}$ gray levels, i.e., $0-15$ gray values, and the binary number of 10 gray values is 1010 . The process of grayscale modulation is equivalent to dividing the 4-bit image into four binary images, in which gray represents the time when the micromirror is on and white represents the time when the micromirror is off. From the lowest significant bit (LSB) to the highest significant bit (HSB) modulation, a frame is divided into four separate bit time slices. The length of time slices is proportional to the binary weight of the bit. When the HSB is allocated to $8 / 15$ frames, HSB- 1 is allocated to $4 / 15$ frames, HSB-2 is allocated to $2 / 15$ frames, and LSB is allocated to $1 / 15$ frames. The binary " 1 " of the PWM control signal will drive the DMD to the "on" position, while " 0 " will make the DMD move to the "off" position. In HSB time slice and HSB-2 time slice, the DMD is in "on" state, while in HSB-1 time slice and LSB time slice, the 
DMD is in "off" state. After the integration of this display sequence, the modulation effect equivalent to gray value 10 is produced, so four binary images are superimposed on the display screen to complete the display of this 4-bit image. 8-bit and 16-bit images can also be implemented, which mainly depends on the reset time of the lens and the field time of each frame of image.

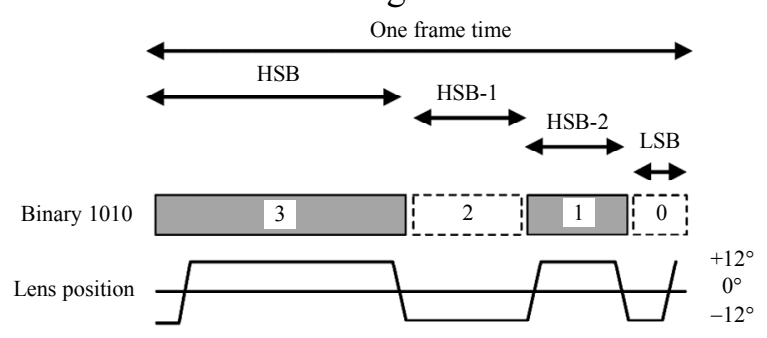

Fig. 2 Schematic diagram of 4-bit pulse width modulation. Pulse width modulation with 1010 binary image data, in which gray represents the time when the micromirror is on and white represents the time when the micromirror is off.

If the gray level of an image is $N$, the display of each grayscale image in the DMD can be divided into $n=\log _{2} N$ number of time slices of data bits. The image is converted into $n$ binary black and white images, and $f(x, y)$ represents a grayscale image, then:

$$
f(x, y)=\sum_{i=1}^{n} 2^{i-1} b_{i}(x, y)
$$

where $b_{i}(x, y)$ is the binary image displayed by the DMD within the $i$ th number of time slices of data bits. Therefore, adjusting the DMD display frame rate is equal to adjusting the stabilization time of the micromirrors. In summary, the process of adjusting the display frequency of the DMD is the process of modulating the grayscale of the DMD, that is, controlling the PWM.

\section{Control module and timing design based on FPGA}

The scheme adopts random measurement matrices with the gray level. We design a high-precision control and counting module based on the FPGA, which can control the DMD working in the external synchronization mode to load random measurement matrices sequentially and record the number of photons of each frame synchronously, that is, the measured value synchronously.

The control and counting module based on the FPGA is implemented on the Cyclone IV chip of Altera DE2-115 development board. The main working sequence of synchronous control pulse is shown in Fig. 3. The sampling frequency pulse is generated by the FPGA, and the sampling frequency pulse signal period is consistent with the time of the last pulse control signal. The number of the gated square wave signal is consistent with the random measurement matrix, and the corresponding control pulse rising edge in each square wave is aligned with the sampling frequency pulse signal generated by dividing frequency. The number is equal to the preset sampling and the interval between each pulse is proportional distribution. When the number of gated square-wave control pulses is 3 in Fig. 3, the control signal pulse time interval is $T 1: T 2: T 3=4: 2: 1$. When a rising edge of this synchronization pulse signal is received, a random binary matrix is loaded into the DMD, and the measured data can be obtained by the PMT. When the next synchronization pulse signal is coming, the measured data and random binary matrix are sent to personal computer (PC) for image reconstruction by CS algorithm.

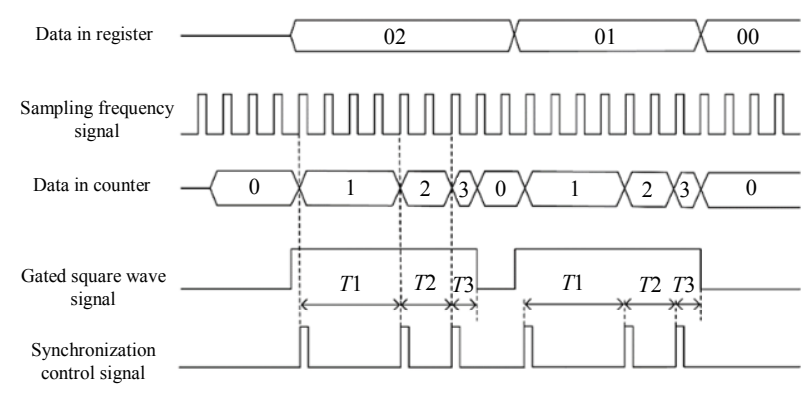

Fig. 3 Diagram of generated synchronization control signal sequence based on FPGA. The proportion distribution of time interval is $T 1: T 2: T 3=4: 2: 1$.

The numerical octal random matrix based on the transformation of the Gaussian matrix is shown in Fig. 4, and the values of some elements without numbers are 0 . In this scheme, the measurement matrix is loaded on the DMD with a size of $1024 \times 768$. The random measurement matrix is 
composed of $M \times N$ matrix elements, and the elements of each matrix are no longer " 0 " or " 1 ", but also have other numbers. Therefore, the DMD micromirror arrays corresponding to a matrix element are grouped together as one pixel. In this scheme, if the value of the representative measurement matrix coefficient is higher, the display time on the corresponding DMD pixel position is longer, and the coefficient value is proportional to the display time.

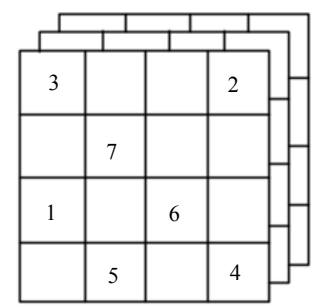

Fig. 4 Octal Gaussian random measurement matrix. The value of matrix elements ranges from 0 to 7 , in which the element corresponding to the part not marked with value is 0 .

Taking the octal Gaussian random matrix of Fig. 4 as an example, the process of independent representation of the corresponding numerical matrix is shown in Fig. 5. First, the octal numerical correspondence in the matrix is transformed into a binary representation. Then, the corresponding high-to-low-bit elements are taken out sequentially, and three measurement matrices are separately obtained.
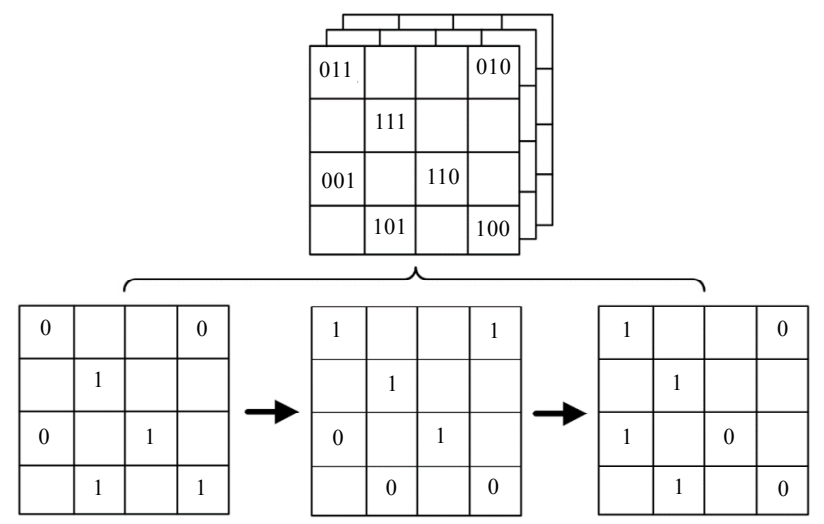

Fig. 5 Representation of numerical matrices independently. The elements in the octal Gaussian random matrix are expressed in binary, and then three measurement matrices are separated from the high to the low, which are sequentially represented.

Under the synchronous control pulse signal of the FPGA, three measurement matrices are displayed from high to low sequentially, and the display time is determined by the synchronous control pulse signal.

\section{Detection limit and experimental performance verification}

We assume that the image is $K$-sparse, and the average photon count rate reflected by the micromirror is $R_{\mathrm{ph}}$ cps. If the measurement time is $t$ for the imaging target, the measured photon count is

$$
W_{\mathrm{ph}}=R_{\mathrm{ph}} t .
$$

The pulse count output by the detector is the sum of the signal photon number $W_{\mathrm{ph}}$, the background photon count $W_{b}$, and the dark count $W_{d}$. As the illumination on the object is at the single photon level, the measurement noise in our imaging system is dominated by Poisson shot noise, and the total noise component can be analyzed as follows:

$$
w^{2}=\sqrt{W_{\mathrm{ph}}+W_{b}+W_{d}}+\sqrt{W_{b}+W_{d}} .
$$

We assume that the background photon count rate is $R_{m}$ and the dark count rate is $R_{n}$. The $S N R$ of single photon imaging system will be

$$
\begin{aligned}
S N R=\frac{W_{\mathrm{ph}}}{w} & =\frac{R_{\mathrm{ph}} t}{\sqrt{R_{\mathrm{ph}} t+2\left(R_{m} t+R_{n} t\right)}} \\
& =\frac{R_{\mathrm{ph}} \sqrt{t}}{\sqrt{R_{\mathrm{ph}}+2\left(R_{m}+R_{n}\right)}} .
\end{aligned}
$$

In the photon counting mode, if we define the detection limit as the light level where the $S N R$ equals to 1 and the measurement time is one second, the photon counting rate at the detection limit can be approximately expressed as

$$
R_{\mathrm{ph}}=\frac{1+\sqrt{1+8\left(R_{m}+R_{n}\right)}}{2} \approx \sqrt{2\left(R_{m}+R_{n}\right)} .
$$

Through the actual system experiment, the photon counting rate range is $10^{2}$ counts/s$10^{3}$ counts/s. To compare the reconstruction performance of different matrices more accurately, the photon counting rate of the detector is used to represent the intensity of light. The photon counting rate at the light intensity level is approximately 
300 counts/s. The image pixels in the experimental system are $64 \times 64$, and only 13756 detected photons are contained in the measured data, corresponding to 3.36 photons per pixel.

In the process of compressed sensing, the performance of the measurement matrix is closely related to the sparsity ratio of the matrix and the compressive sampling ratio (SR). The performance of the measurement matrix can be evaluated by the reconstruction quality of image. For a quantitative comparison of the image quality, we introduce three commonly used full-reference evaluation indexes, namely, the mean square error (MSE), peak signal to noise ratio (PSNR), and mean structure similarity index (MSSIM). In order to evaluate the resolution of the reconstructed image, the customized mask pattern is used as the imaging target of the experimental system. The image reconstruction algorithm is implemented by the TVAL3 algorithm.

\subsection{Influence of sparsity ratio on imaging quality}

Because this scheme adopts random measurement matrix with the gray level, the influence of sparsity ratio of the measurement matrix on matrix performance is studied through simulation experiments. Taking the construction of quaternary Gaussian random matrix as an example, the sparsity ratio of the matrix is defined as the proportion of non-zero elements of the matrix. In order to obtain multiple sets of data to prove the universality of the results, reconstruction experiments at different compressive SRs are set up. The compressive SRs of the three groups are set to $0.25,0.50$, and 0.75 , respectively, and the matrix sparsity ratio of each group increases continuously.

The experimental results are shown in Fig. 6. In order to obtain the simulation results of the measurement matrix under different sparsity ratios, Figs. 6(a), 6(b), and 6(c) correspond to the indexes $M S E, P S N R$, and MSSIM, respectively, and each data point has an average of 500 independent simulation experiments. We can see that the quality of gray image reconstruction will increase with an

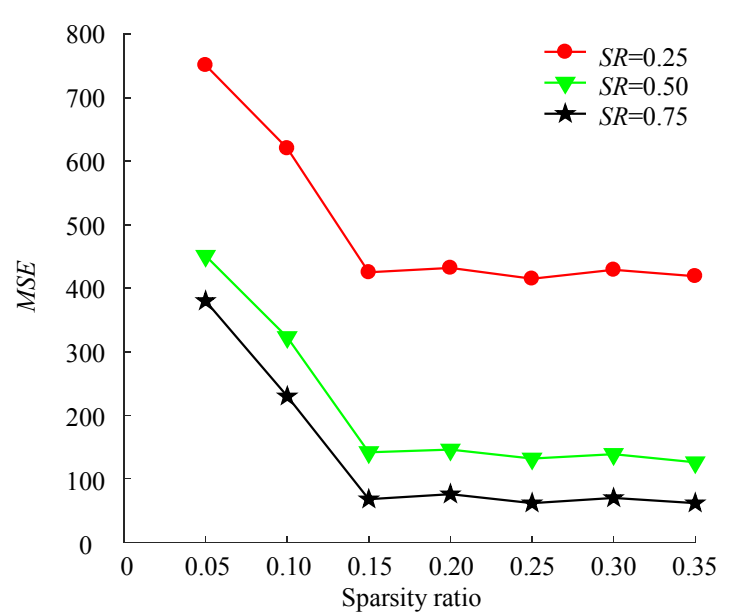

(a)

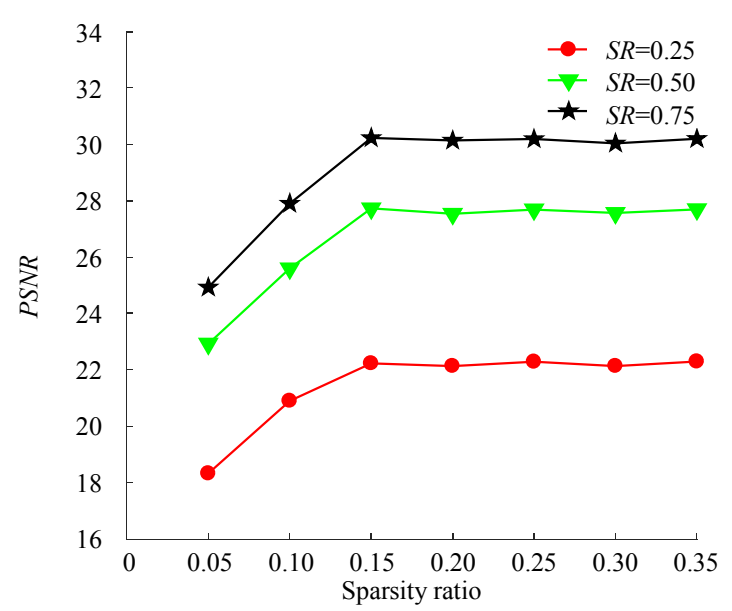

(b)

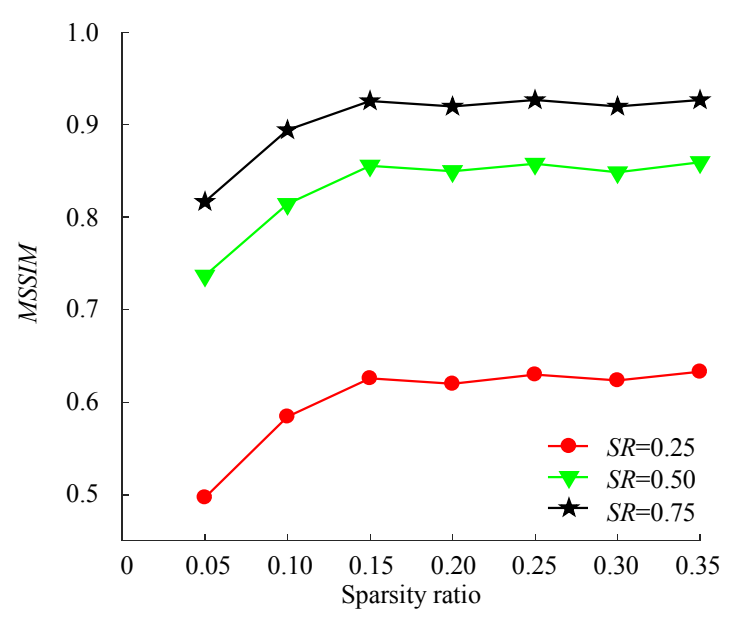

(c)

Fig. 6 Effect of matrix sparsity ratio on its performance. With $S R S$ of $0.25,0.50$, and 0.75 , the parameters $M S E$ (a), PSNR (b), and MSSIM (c) change with sparsity ratio of the measurement matrix. Each group of experiments is repeated 500 times. 
increase in compressive $S R$, and the quality of the reconstructed gray image is closely related to the matrix sparsity ratio. When the sparsity ratio of matrix is small, the quality of the reconstructed image will increase with an increase in the matrix sparsity ratio. But for a larger sparsity ratio (greater than 0.15 ), the reconstruction quality stays almost unchanged. Therefore, there is a tradeoff between the matrix sparsity ratio and the compressive $S R$. When the matrix sparsity ratio is greater than 0.15 , the $S R$ will be increased as much as possible to improve the quality of gray image reconstruction. However, in the actual single photon compressive imaging experiment, while reducing the sparsity ratio of the matrix, sufficient luminous flux should be guaranteed to obtain a higher $S N R$.

In order to validate the above conclusions, we use custom mask of patterns as imaging targets. The compressive $S R$ s of three groups of experiments shown in Figs. 7(a), 7(b), and 7(c) are set to 0.25, 0.50 , and 0.75 , and the sparsity ratio of each group is increased in turn. Three groups of experiments are set to $0.05,0.10$, and 0.15 , respectively, and Fig. 7 shows the experimental results of the quaternary Gaussian random matrix under these conditions. The first group of experiments shown in Figs. 7(a1) 7(a3) represents the reconstruction results of increasing the sparsity ratio of the matrix sequentially when the compressive $S R$ is 0.25 . The second group of experiments shown in Figs. 7(b1) 7(b3) represents the reconstruction result of increasing the sparsity ratio of the matrix sequentially when the compressive $S R$ is 0.50 . The third group of experiments shown in Figs. 7(c1) 7(c3) represents the reconstruction result of increasing the sparsity ratio of the matrix sequentially when the compressive $S R$ is 0.75 . As can be seen from Fig. 7, the quality of the reconstructed image is improved with an increase in matrix sparsity ratio.

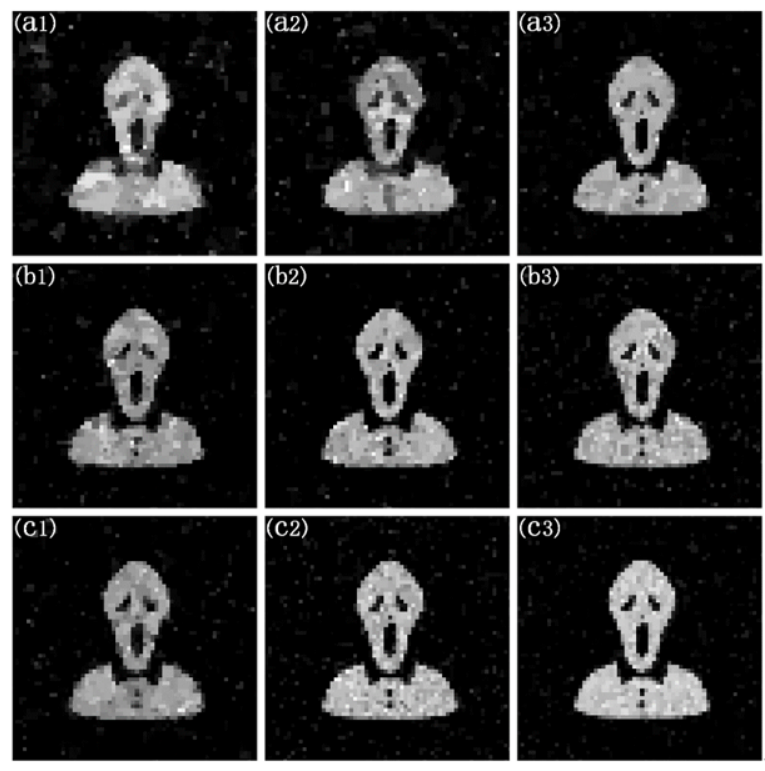

Fig. 7 Compressive $S R$ s of the three groups of experiments [(a), (b), and (c)] are set to $0.25,0.50$, and 0.75 , respectively. The sparsity ratio of each group is increased in turn, and the sparsity ratios of each group are set to $0.05,0.10$, and 0.15 , respectively.

\subsection{Influence of compressive sampling ratio on imaging quality}

In order to analyze the influence of the number of measurements on the quality of image reconstruction, we set different compressive $S R$ s to verify the corresponding experiments results.

Gaussian binary random matrix, quaternary Gaussian random matrix, octal Gaussian random matrix, and hexadecimal Gaussian random matrix are used as measurement matrices for compressed sampling and image reconstruction. At the same time, the orthogonal matrix of partial Fourier is used for the experiment by contrast.

The imaging results corresponding to the above five random matrices are shown in Fig. 8. The pattern of the flower is used as the imaging target of the experimental system corresponding to the Gaussian binary random matrix. Taking the Chinese character pattern of "light" as the imaging target, it corresponds to the quaternary Gaussian random matrix, and the United States Air Force (USAF) resolution test board is selected as the imaging target of the experimental system corresponding to the octal Gaussian random matrix. The "aircraft" pattern 
is taken as the imaging target of the experimental system corresponding to the hexadecimal Gaussian random matrix. The pattern of the "triangle combination" is used as the imaging target of the experimental system corresponding to the hexadecimal Fourier matrix. Among them, Figs. 7(a1) $-7(\mathrm{a} 4), 7(\mathrm{~b} 1)-7(\mathrm{~b} 4), 7(\mathrm{c} 1)-7(\mathrm{c} 4)$, $7(\mathrm{~d} 1)-7(\mathrm{~d} 4)$, and $7(\mathrm{e} 1)-7(\mathrm{e} 4)$ correspond to the above five experiments of image reconstruction. In a single group of the above five experiments, there are four independent groups with corresponding compressive $S R$ s of $0.10,0.25,0.50$, and 0.75 , respectively. The sparsity ratio of the selected measurement matrix is 0.15 , and the imaging resolution is $64 \times 64$. As can be seen from Fig. 8, based on the compressed sensing theory, it can recover the original signal from a small number of measurements, and with an increase in the number of measurements, the quality of image reconstruction becomes better and better.
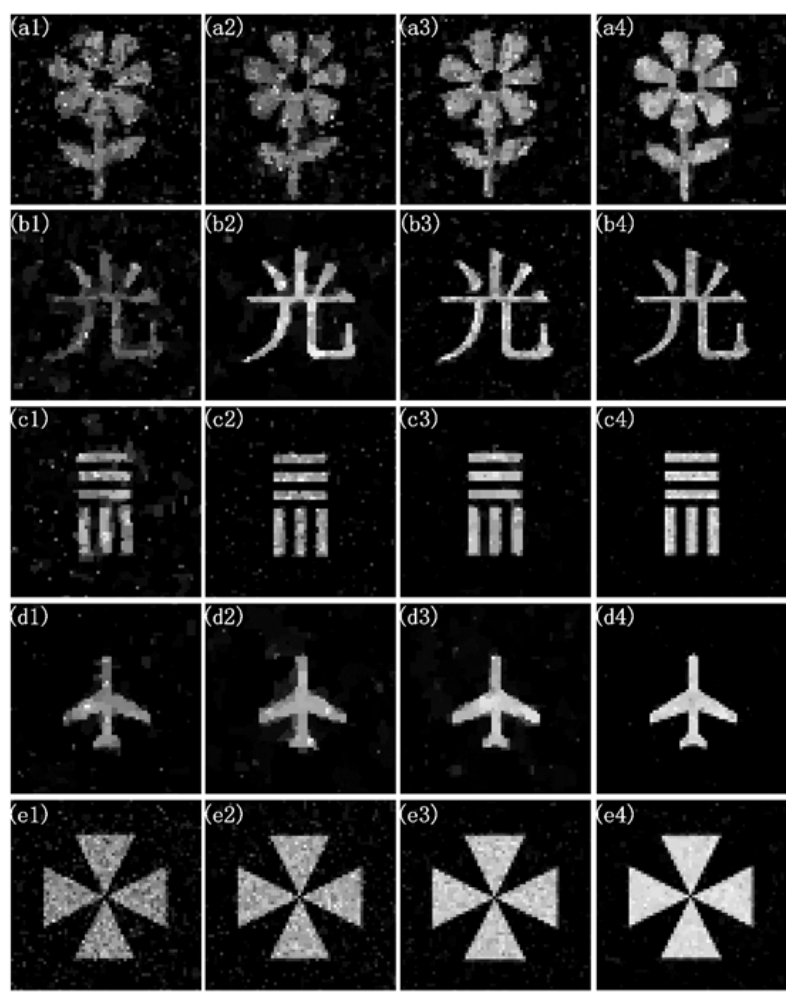

Fig. 8 Reconstruction results of five groups of random matrices at corresponding compressive $S R$. In a single group of five experiments, the compressive $S R$ s of the four independent groups are set to $0.10,0.25,0.50$, and 0.75 , respectively.
In order to compare the quality of the reconstructed image of the imaging object in the experimental system more intuitively, the Gaussian gray matrix and the Fourier gray matrix are evaluated by the MSE, PSNR, and MSSIM. The reconstructed image indexes change with the compressive $S R \mathrm{~s}$ as shown in Fig. 9. From the MSE, $P S N R$, and MSSIM evaluation indices corresponding to the reconstructed gray images, it can be seen that when the compressive $S R$ is less than 0.40 , the reconstruction performance of the three gray-scale matrices is relatively poor, while when the compressive $S R$ is greater than 0.40 , the performance of the random matrices is improved with an increase in the compressive $S R$. In addition, when the compressive $S R$ is close to 0.10 and 0.75 , the reconstruction performance of the three gray-scale matrices has little difference.

Compared with the Gaussian gray matrix, the Fourier gray matrix has better performance. And with an increase in the gray level of gray matrix, the reconstruction performance of these two kinds of gray matrices is much better than that of binary matrices.

\subsection{Comparison of imaging performance of different measurement matrices}

The performances of different types of measurement matrices are analyzed and verified by simulation experiments. In order to obtain the evaluation indexes of reconstructed images under different measurement matrices, the simulation results are averaged for 1000 independent experimental results to reduce the impact of randomness on experimental results. The compressive $S R$ is 0.50 , and the sparsity ratio is 0.15 . Table 1 presents the reconstruction results of the $M S E, P S N R$, and MSSIM of the reconstructed image under the same experimental conditions. From the experimental results in Table 1, we can see that the hexadecimal Gaussian random matrix has the best performance, and the MSSIM is 0.9003 , followed by the octal Gaussian random matrix. 


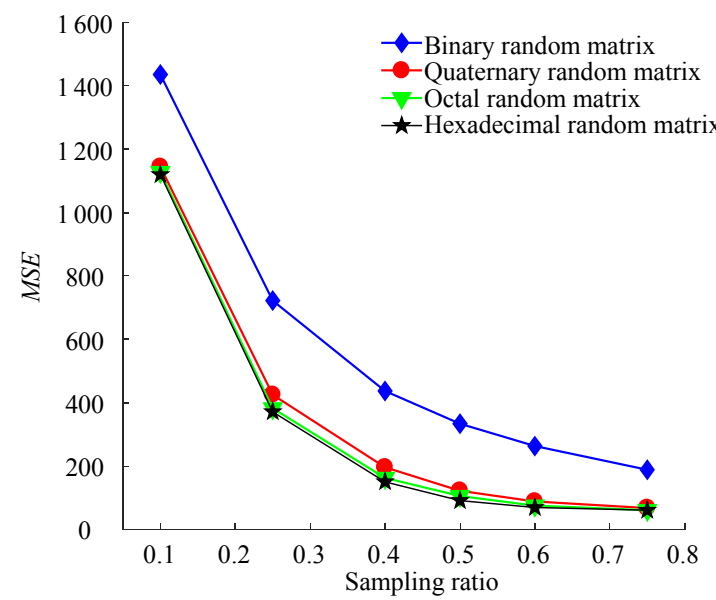

(a)

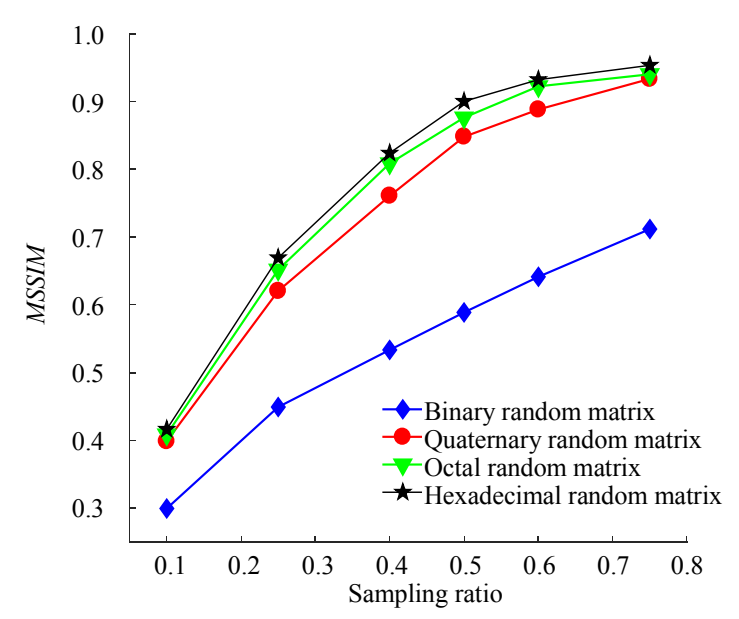

(c)

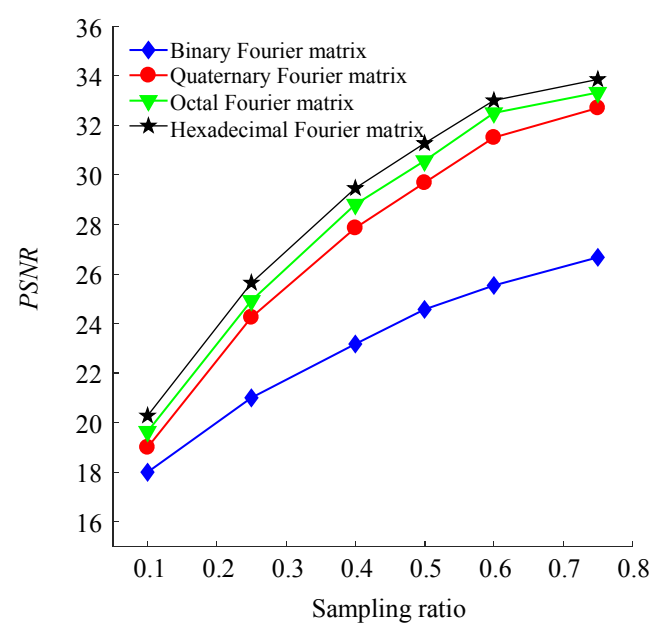

(e)

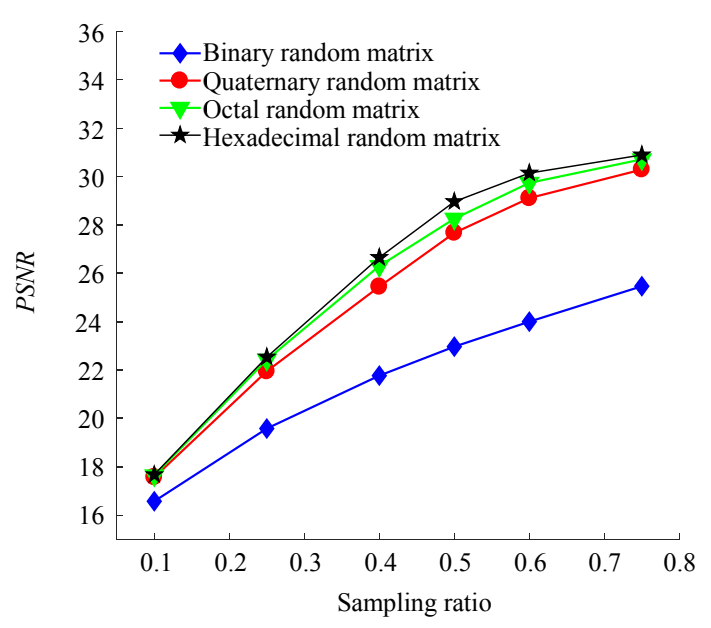

(b)

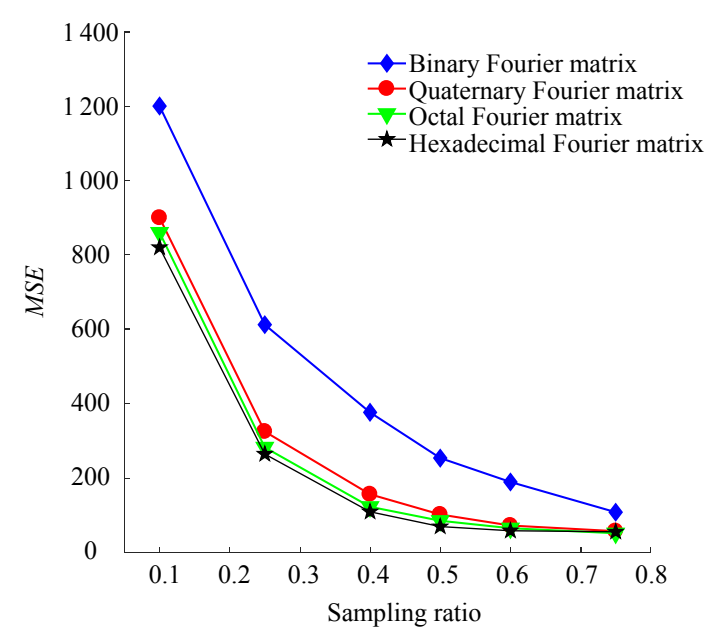

(d)

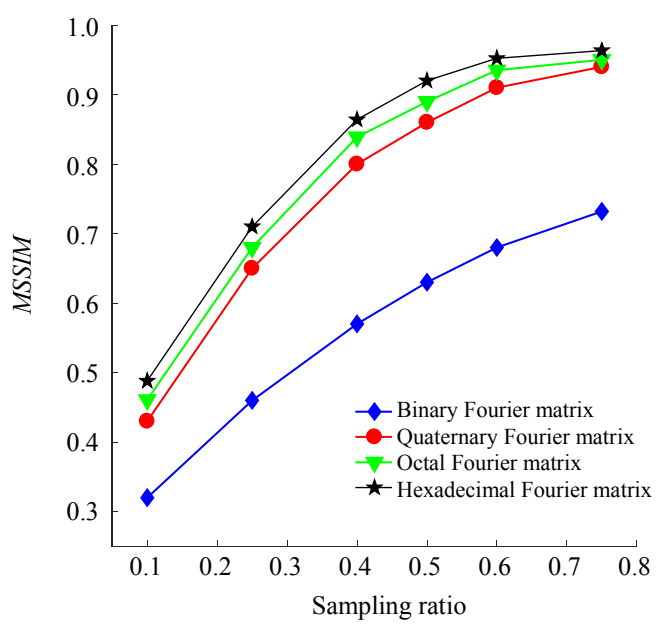

(f)

Fig. 9 Corresponding curves of the evaluation indices of compressive $S R$ change. (a) and (d) represent the $M S E$ of the reconstructed image with the changing compressive $S R$; (b) and (e) represent the $P S N R$ of the reconstructed image with the changing compressive $S R$; (c) and (f) represent the $M S S I M$ of the reconstructed image with the changing compressive $S R$. 
Table 1 Evaluation indices of reconstruction image under different measurement matrices.

\begin{tabular}{cccc}
\hline Evaluation index & $M S E$ & $P S N R$ & $M S S I M$ \\
\hline $\begin{array}{c}\text { Quaternary Gaussian random } \\
\text { matrix }\end{array}$ & 122.4975 & 27.6769 & 0.8481 \\
$\begin{array}{c}\text { Octal Gaussian random } \\
\text { matrix }\end{array}$ & 105.2289 & 28.2637 & 0.8762 \\
$\begin{array}{c}\text { Hexadecimal Gaussian } \\
\text { random matrix }\end{array}$ & 91.5916 & 28.9704 & 0.9003 \\
\hline
\end{tabular}

\section{Conclusions}

In this paper, a digital grayscale modulation method is proposed to improve the efficiency of compressive sampling and a single photon compressive imaging system is established. With this system, we realize the reconstruction of the gray images by digital grayscale modulation of the DMD based on gray-scale matrices, which are improved based on the Gaussian random matrix. The control and photon counting module are designed based on the FPGA independently. This paper analyzes and constructs new gray-scale measurement matrices. The quality of gray image reconstruction is compared among three kinds of gray matrices and binary matrix, and the gray matrix has better performance than binary matrix. In addition, the quality of image reconstruction can be improved by reducing the sparsity ratio of the matrix appropriately and increasing the compressive $S R$. In order to obtain better image reconstruction quality under conditions of limited acquisition and the sparsity ratio of the matrix, the sparsity ratio is preferably 0.15 , and the compressive $S R$ is preferably 0.50 . The experimental results show that the image reconstruction performance of the gray matrix with a higher gray level is better.

\section{Acknowledgment}

This work was supported in part by the National Natural Science Foundation of China (Grants Nos. 61865010 and 61565012), in part by the China Postdoctoral Science Foundation (Grant No. 2015T80691), in part by the Science and Technology Plan Project of Jiangxi Province
(Grant No. 20151BBE50092), and in part by the Funding Scheme to Outstanding Young Talents of Jiangxi Province (Grant No. 20171BCB23007).

Open Access This article is distributed under the terms of the Creative Commons Attribution 4.0 International License (http://creativecommons.org/licenses/by/4.0/), which permits unrestricted use, distribution, and reproduction in any medium, provided you give appropriate credit to the original author(s) and the source, provide a link to the Creative Commons license, and indicate if changes were made.

\section{References}

[1] R. S. Bennink, S. J. Bentley, R. W. Boyd, and J. C. Howell, "Quantum and classical coincidence imaging," Physical Review Letters, 2004, 92(3): 033601 .

[2] Q. R. Yan, H. Wang, C. L. Yuan, B. Li, and Y. H. Wang, "Large-area single photon compressive imaging based on multiple micro-mirrors combination imaging method," Optics Express, 2018, 26(15): 19080-19090.

[3] W. K. Yu, X. F. Liu, X. R. Yao, C. Wang, S. Q. Gao, G. J. Zhai, et al., "Single photon counting imaging system via compressive sensing," Preprint arXiv, 2012: 1202.5866.

[4] Y. S. Zhang, Y. Xiang, L. Y. Zhang, L. X. Yang, and J. Zhou, "Efficiently and securely outsourcing compressed sensing reconstruction to a cloud," Information Sciences, 2019, 496(1): 150-160.

[5] D. Liu, Q. S. Wang, Y. Zhang, X. Liu, J. Lu, and J. Sun, "FPGA-based real-time compressed sensing of multichannel EEG signals for wireless body area networks," Biomedical Signal Processing and Control, 2019, 49(1): 221-230.

[6] Z. Cui, J. L. Yang, S. D. Jiang, J. Li, L. Lin, and Y. $\mathrm{Gu}$, "An infrared-small-target detection method in compressed sensing domain based on local segment contrast measure," Infrared Physics \& Technology, 2018, 93(1): 41-52.

[7] W. Becker, A. Bergmann, M. A. Hink, K. König, K. Benndorf, and C. Biskup, "Fluorescence lifetime imaging by time-correlated single-photon counting," Microscopy Research and Technique, 2004, 63(1): 58-66.

[8] J. Romberg, "Imaging via compressive sampling," IEEE Signal Processing Magazine, 2008, 25(2): 14-20.

[9] M. F. Duarte, M. A. Davenport, D. Takhar, J. N. Laska, T. Sun, K. F. Kelly, et al., "Single-pixel imaging via compressive sampling," IEEE Signal Processing Magazine, 2008, 25(2): 83-91. 
[10] R. G. Baraniuk, "Compressive sensing [lecture notes]," IEEE Signal Processing Magazine, 2007, 24(4): 118-121.

[11] Q. Tong, Y. L. Jiang, H. Y. Wang, and L. Guo, "Image reconstruction of dynamic infrared single-pixel imaging system," Optics Communications, 2018, 410: 35-39.

[12] Z. Zhang, X. Ma, and J. Zhong, "Single-pixel imaging by means of Fourier spectrum acquisition," Nature Communications, 2015, DOI: $10.1038 /$ ncomms 7225 .

[13] K. Taguchi and J. S. Iwanczyk, "Vision 20/20: Single photon counting $\mathrm{x}$-ray detectors in medical imaging," Medical Physics, 2013, DOI: 10.1118/1.4820371.

[14] Y. Chen and Y. Chi, "Robust spectral compressed sensing via structured matrix completion," IEEE Transactions on Information Theory, 2014, 60(10): 6576-6601.

[15] M. J. Sun and J. M. Zhang, "Single-pixel imaging and its application in three-dimensional reconstruction: a brief review," Sensors, 2019, DOI: 10.3390/s19030732.

[16] M. J. Sun, M. P. Edgar, G. M. Gibson, B. Sun, N. Radwell, R. Lamb, et al., "Single-pixel three-dimensional imaging with time-based depth resolution," Nature Communications, 2016, 7(1): 429-430.

[17] B. Sun, M. P. Edgar, R. Bowman, L. E. Vittert, S. Welsh, A. Bowman, et al., "3D computational imaging with single-pixel detectors," Science, 2013, 340(6134): 844-847.

[18] Y. Xiao, W. L. Gao, G. H. Zhang, and H. Zhang, "Compressed sensing based apple image measurement matrix selection," International Journal of Distributed Sensor Networks, 2015, 11(7): 5862-5875.

[19] Y. Yu, A. P. Petropulu, and H. V. Poor, "Measurement matrix design for compressive sensing based MIMO radar," IEEE Transactions on Signal Processing, 2011, 59(11): 5338-5352.

[20] V. Tiwari, P. P. Bansod, and A. Kumar, "Designing sparse sensing matrix for compressive sensing to reconstruct high resolution medical images," Cogent Engineering, 2015, DOI: 10.1080/23311916.2015. 1017244.

[21] H. Nouasria and M. Et-tolba, "New constructions of Bernoulli and Gaussian sensing matrices for compressive sensing," in 2017 International Conference on Wireless Networks and Mobile Communications (WINCOM), Morocco, December 25, 2017, pp. 1-6.

[22] J. Ding, D. Bao, Q. Wang, X. He, H. Bai, and S. Li, "A novel multi-dictionary framework with global sensing matrix design for compressed sensing," Signal Processing, 2018, 152(1): 69-78.

[23] W. K. Yu, X. F. Liu, X. R. Yao, C. Wang, G. J. Zhai, and Q. Zhao, "Single-photon compressive imaging with some performance benefits over raster scanning," Physics Letters A, 2014, 378(45): 34063411.

[24] X. F. Liu, W. K. Yu, X. R. Yao, B. Dai, L. Z. Li, C. Wang, et al., "Measurement dimensions compressed spectral imaging with a single point detector," Optics Communications, 2016, 365: 173-179.

[25] E. J. Candes, "The restricted isometry property and its implications for compressed sensing," Comptes Rendus Mathematique, 2008, 346(9): 589-592.

[26] Y. T. Chen and J. G. Peng, "Influences of preconditioning on the mutual coherence and the restricted isometry property of Gaussian/Bernoulli measurement matrices," Linear and Multilinear Algebra, 2016, 64(9): 1750-1759.

[27] H. Monajemi, S. Jafarpour, M. Gavish, Stat 330/CME 362 Collaboration, and D. L. Donoho, "Deterministic matrices matching the compressed sensing phase transitions of Gaussian random matrices," Proceedings of the National Academy of Sciences, 2013, 110(4): 1181-1186.

[28] T. Huang, Y. Z. Fan, and M. Hu, "Compressed sensing based on random symmetric Bernoulli matrix," in 2017 32nd Youth Academic Annual Conference of Chinese Association of Automation (YAC), China, May 19-21, 2017, DOI: 10.1109/YAC.2017.7967403.

[29] D. Dudley, W. M. Duncan, and J. Slaughter, "Emerging digital micromirror device (DMD) applications," Proceedings of SPIE, 2003, DOI: f10.1117/12.480761.

[30] K. Zhang, Y. Huang, J. Yan, and L. Sun, "Dynamic infrared scene simulation using grayscale modulation of digital micro-mirror device," Chinese Journal of Aeronautics, 2013, 26(2): 394-400.

[31] R. Höfling and E. Ahl, "ALP: Universal DMD controller for metrology and testing," Proceedings of SPIE, 2004, DOI: 10.1117/12.528336. 